\title{
Crystal structure of human MTH1 and the 8-0xo-dGMP product complex
}

\author{
Linda M Svensson ${ }^{1 \#}$, Ann-Sofie Jemth ${ }^{2 \#}$, Matthieu Desroses², Olga Loseva ${ }^{2}$, \\ Thomas Helleday $^{2}$, Martin Högbom ${ }^{1}$, Pål Stenmark ${ }^{*}$
}

\footnotetext{
${ }^{1}$ Department of Biochemistry and Biophysics, Stockholm University, SE-106 91 Stockholm, Sweden. ${ }^{2}$ Department of Genetics, Microbiology and Toxicology, Stockholm University, SE-106 91 Stockholm, Sweden.
}

\begin{abstract}
*To whom correspondence should be addressed. Department of Biochemistry and Biophysics, Stockholm University, SE-106 91 Stockholm, Sweden. Fax: +46 8 153679, Tel: +46 8 162110, E-mail: stenmark@dbb.su.se
\end{abstract}

\# These authors contributed equally to this work.

\begin{abstract}
MTH1 hydrolyses oxidized nucleotide triphosphates, thereby preventing them from being incorporated into DNA. We here present the structures of human MTH1 (1.9 $\AA)$ and its complex with the product 8 -oxo-dGMP $(1.8 \AA)$. Unexpectedly MTH1 binds the nucleotide in the anti conformation with no direct interaction between the 8-oxo group and the protein. We suggest that the specificity depends on the stabilization of an enol tautomer of the 8-oxo form of dGTP. The binding of the product induces no major structural changes. The structures reveal the mode of nucleotide binding in MTH1 and provide the structural basis for inhibitor design.
\end{abstract}




\section{Introduction}

Oxygen radicals are produced as a byproduct of cellular metabolism. Reactive oxygen species (ROS) cause damage not only to the DNA directly, but also to the free bases in the nucleotide pool, resulting in for instance 8-oxo-dGTP, which can be misincorporated into the DNA and if unrepaired can cause mutations in the second round of replication by misparing with thymine. The MTH1 (MutT Homolog 1) protein effectively degrades oxidized nucleotides, by catalyzing for instance the hydrolysis of 8-oxo-dGTP to 8-oxodGMP and thereby preventing their incorporation into DNA. The MTH1 protein belongs to the Nudix hydrolase superfamily, characterized by a conserved 23-residue sequence segment, $\left(\mathrm{GX}_{5} \mathrm{EX}_{7} \mathrm{REUXEEXGU,} \mathrm{U}=\mathrm{I}, \mathrm{L}\right.$ or $\left.\mathrm{V}\right)$. A solution structure of MTH1 has previously been reported [1]. MTH1 is present both in the nucleus and the mitochondria where it also minimizes accumulation of 8-oxo-guanine in DNA [2]. Indeed, MTH1-/knockout mice or cells siRNA depleted for MTH1 accumulate 8-oxo-G in their DNA and display a slight increase in mutation and cancer rates [3-5]. MTH1 also accepts other substrates such as 2-OH-dATP, 2-OH-rATP and 8-OH-dATP [6,7]. The production of the mono-phosphate, as opposed to the di-phosphate, is likely important to prevent regeneration of 8-oxo-dGTP by nucleotide di-phosphate kinase [8]. Effective supply of dGTP is essential for DNA replication. Hence, it is of interest how the MTH1 structurally recognize and distinguish the 8-oxo-dGTP from dGTP. Here, we have resolved the crystal structure of human MTH1 and report a distinct mechanism for recognition, different from that of the E.coli MutT homolog. 


\section{Materials and Methods}

\subsection{MTH1 Expression, purification and activity assay}

cDNA encoding human MTH1 (NUDT1) and optimized for expression in E. coli was purchased from Geneart AG. His tagged MTH1 was expressed from pET-28a (Novagen) in E. coli BL-21(DE3) (Stratagene). The bacteria were harvested, lysed using BugBuster (Novagen) and His tagged MTH1 was affinity purified using HisTrap HP (Amersham Biosciences). The His tag was removed by thrombin digestion (Novagen) and the MTH1 protein further purified using anion exchange chromatography at $\mathrm{pH} 7.5$ using a MonoQ column (GE Healthcare). The identity and purity of the protein was confirmed using MALDI-TOF mass spectrometry. The activity of MTH1 was monitored using 8-oxodGTP (TriLink Biotechnologies) using the PPiLight ${ }^{\mathrm{TM}}$ Inorganic Pyrophosphate Assay (Lonza Rockland, Inc.) The enzyme activity was calculated using linear regression and $\mathrm{k}_{\mathrm{cat}}, \mathrm{K}_{\mathrm{M}}$ and $\mathrm{k}_{\mathrm{cat}} / \mathrm{K}_{\mathrm{M}}$ were determined by fitting the data points to the Michaelis Menten equation using non-linear regression analysis utilizing the GraphPad Prism Software.

\subsection{Crystallization}

$3 \mathrm{mM}$ 8-oxo-dGTP, $6 \mathrm{mM} \mathrm{MgCl} 2$ and $2 \mathrm{mM}$ TCEP was added to MTH1 (16.9 $\mathrm{mg} / \mathrm{ml})$ in $20 \mathrm{mM}$ TrisHCl pH 7.4, $0.15 \mathrm{M} \mathrm{NaCl}$ and $5 \%$ glycerol. A hanging drop experiment was set up, where $100 \mathrm{nl}$ protein solution was mixed with $100 \mathrm{nl}$ well solution $(26 \%$ PEG6000, 0.1 M Sodium Acetate Trihydrate $\mathrm{pH}$ 4.0, 0.2 $\mathrm{M} \mathrm{Li}_{2} \mathrm{SO}_{4}$ ). The plate was stored at $20^{\circ}$ C. A long, thin, rod-like crystal was flash frozen in liquid nitrogen without adding an additional cryo protectant to the crystallization buffer. Apo MTH1 $(8.5 \mathrm{mg} / \mathrm{ml})$ in 
buffer completed with $2 \mathrm{mM}$ DTT replacing TCEP was crystallized as the 8-oxo-dGMP complex above. Crystals appeared at the same well solution conditions as above, the crystal was flash frozen in liquid nitrogen after the addition of cryo solution (27\% PEG6000, $160 \mathrm{mM} \mathrm{Li}_{2} \mathrm{SO}_{4}, 80 \mathrm{mM}$ Sodium Acetate Trihydrate $\mathrm{pH}$ 4.0, $160 \mathrm{mM} \mathrm{NaCl}$ and $15 \%$ glycerol).

\subsection{Structure determination and model building}

Molecular replacement was carried out using a dataset collected at the Diamond synchrotron. The model was built and refined in a higher quality data set collected at 100K at beamline BL14.1, BESSY. Both datasets were processed using MOSFLM and SCALA in the CCP4 program suite [9]. Data collection and refinement statistics are presented in Table 1. Balbes [10] and the template pdb file 3FK9 were used for molecular replacement. ARP/wARP [11] was used for building the initial model, followed by iterative building cycles using Coot [12] and Refmac5 [13], TLS was used. The coordinates and structure factors for the structures presented in this paper were deposited in the PDB under codes 3ZR1 (apo) and 3ZR0 (complex).

\section{Results and Discussion}

\subsection{Enzyme activity}

The activity of the MTH1 protein was tested with 8-oxo-dGTP as substrate. The catalytic parameters obtained were: $\mathrm{K}_{\mathrm{M}} 13.2 \pm 2.5 \mu \mathrm{M}, \mathrm{k}_{\text {cat }} 3.3 \pm 0.3 \mathrm{~s}^{-1}, \mathrm{k}_{\text {cat }} / \mathrm{K}_{\mathrm{M}} 0.25 \pm 0.05 \mu \mathrm{M}^{-1} \mathrm{~s}^{-}$

${ }^{1}$, Figure 1A. The determined $\mathrm{K}_{\mathrm{M}}$ value is in good agreement with previous studies [7]. 
We determined the activity at $22{ }^{\circ} \mathrm{C}$ and $\mathrm{pH} 7.5$ which explains the lower $\mathrm{k}_{\mathrm{cat}} / \mathrm{K}_{\mathrm{M}}$ value obtained compared to the previously published value of $0.81 \mu \mathrm{M}^{-1} \mathrm{~s}^{-1}$ determined at $30^{\circ} \mathrm{C}$ and $\mathrm{pH} 8.0$ [7]. The kinetic analysis shows that the protein preparation used for crystallization experiments is highly active.

\subsection{MTH1 structure and its product complex}

The structure of MTH1 has the typical Nudix fold with an $\alpha / \beta / \alpha$ structure [14] arranged as a mixed $\beta$-sheet with one $\alpha$-helix on each side (Figure 1B). The Nudix motif is located on helix one and connecting loops. The protein crystallized with two monomers in the asymmetric unit. The A chains of the structures include residues 3-156. The B chains include residues 2-156, however residues 14-17 in the B chains were disordered and are not included in the models. In the present crystal form, the B-chain appears less ordered with a slightly worse electron density. The structural description is based on the betterdefined A-chains. The apo structure has an acetate molecule originating from the crystallization buffer bound in the active site. During the preparation of this manuscript an apo structure of human MTH1 was deposited in the PDB (3Q93). The structures are virtually identical; a detailed comparison is available as supplementary information S1.

As expected, when the protein was co-crystallized with the substrate 8-oxo-dGTP, the structure revealed that the reaction had taken place and the product (8-oxo-dGMP) complex structure was obtained. The product base is bound in a pocket formed by the beta sheet and helix two. The product complex shows that MTH1 binds the nucleotide in the anti conformation and not in the syn conformation as previously suggested based on 
nucleotide analog interaction studies [15]. The base in the MTH1 complex is thus oriented in the opposite direction compared to the sugar (Figure 2A). This is in contrast to the MutT protein from E. coli that binds 8-oxo-dGMP in the syn conformation [16] but similar to 8-oxoguanine DNA glycosylase (hOGG1) and MutY [17,18] (Figure 2B). E.coli MutT has been intensively studied and is a founding member of the Nudix family. MutT also catalyses the hydrolysis of 8-oxo-dGTP, but has a low (21\%) sequence identity to MTH1 $[14,19]$.

\subsection{Substrate hydrolysis}

It has been shown that the $\beta$-phosphate of 8 -oxo-dGTP is attacked by water during hydrolysis. Oxygen originating from the solvent water is introduced in the pyrophosphate product [1]. An overlay with the E.coli MutT 8-oxo-dGMP complex reveals that the position of the $\alpha$-phosphate is almost identical between the MTH1 and the MutT complex and the two proteins most likely utilize a conserved site for substrate hydrolysis. Glu100, Glu56 and Glu52 are positioned where they can coordinate a divalent cation needed for activity (Figure 2A). These residues are clustered close to the position of the $\alpha$-phosphate of 8-oxo-dGMP. The product complex was crystallized in presence of $\mathrm{Mg}^{2+}$. Based on coordination geometry, coordination distances and the electron density, magnesium does not appear to be bound to the protein complex. It is however possible that the metal coordinates only to the substrate triphosphate and dissociates upon product formation or binds to the pyrophosphate product. A sequence alignment does not reveal if Asp99 or Glu100 is important for metal coordination. Asp99 points away from the metal binding 
site and it has been shown by mutagenesis that the Asp99Ala mutation retains a high activity while the Glu100Ala mutant is inactive [1]. Glu100 is a metal-coordinating residue located outside the Nudix motif. Lys23 coordinate the $\alpha$-phosphate of 8-oxodGMP.

\subsection{Base recognition}

The active site pocket of MTH1 is built up of residues Leu9, Phe27, Phe72, Met81, Va183, Trp117, Trp123 and Phe139. The base of dGMP makes several hydrogen bonds in the recognition pocket of MTH1. The hydrogen bonds to Asn33, Asp119 and Asp120 appear most important and are discussed below.

The binding of the base is completely different when comparing MTH1 and MutT (Figure 2B) [16]. MTH1 binds 8-oxo-dGMP in the anti conformation and MutT in the syn conformation, an approximately 180 degree difference of the torsion angle about the $\mathrm{N}$-glycosidic bond. The base-protein interactions are completely different and reveal no structural conservation. This reveals that even though the proteins share a conserved catalytic region, the nucleotide recognition is not conserved. Different evolutionary routes have been taken, possibly to allow for the broader substrate specificity of MTH1. 8-oxo-dGMP binding induces a large loop movement in MutT that is critical for recognition [16]. 8-oxo-dGMP binding in MTH1, on the other hand, induces no significant structural changes and the active site of MTH1 appears to be prearranged for substrate binding. The rmsd between the apo and complex structure is merely $0.2 \AA$; they are thus practically identical. 
Trp117, pi stacking with the product base

Both Trp 117 and Trp 123 of MTH1 have been shown by tryptophan fluorescence to be close to the nucleotide binding site [20]. This is confirmed by the crystal structure that places these residues within approximately $3.5 \AA$ of 8-oxo-dGMP. Trp117 pi-stacks with the base of 8-oxo-dGMP and is essential in building up the substrate binding pocket of MTH1. The Trp117Ala mutant does not hydrolyze either 8-oxo-dGTP or 2-OH-dATP while the Trp117Tyr has activity towards 2-OH-dATP [21]. The integrity of the two rings of the nucleotide base are important for binding, probably because of the observed ring stacking [22].

\section{Phe27}

The product complex reveals that Phe27 directly interacts with the product. The Phe27Ala mutation has been shown to reduce the activity of MTH1 to 2-OH-dATP and 8-oxo-dGTP, the interaction with the product explains the lower activity of the Phe27Ala mutation [21].

\section{Asn33, important for specificity}

Asn33 has previously been shown by mutagenesis to be critical for substrate binding and specificity [1]. The nitrogen of Asn33 is hydrogen bonded to N3 of 8-oxo-dGMP, a good hydrogen bond acceptor (2.8 $\AA$ ) (Figure 2A). The oxygen of Asn33 accepts one hydrogen bond from the 2-amino group (2.8 $\AA$ ). Asn33 is critical to substrate binding and is central for the interaction. Mutating Asn33 to Glu abolishes the ability to hydrolyze 8-oxo-dGTP 
[1]. The Asn33Glu mutation introduces a larger side chain and the 8-oxo-dGMP complex shows that this would sterically block 8-oxo-dGTP binding. The Asn33Ala mutation retains $14 \%$ activity with 8 -oxo-dGTP [1].

Asp119, important for specificity

Asp119 forms one hydrogen bond with the 6-oxo group $(2.4 \AA)$ of the base. Its involvement in substrate binding is also observed in the activities of the Asp119Ala and Asp119Asn protein variants. The mutants retain approximately half activity towards 8oxo-dGTP but have almost no activity towards $2-\mathrm{OH}-\mathrm{dATP}[1,21]$. This indicates that the carboxyl group of Asp119 is crucial for the interaction with 2-OH-dATP. Asp119 makes a direct hydrogen bond with the 6-oxo group in the 8-oxo-dGMP complex. The corresponding group in 2-OH-dATP is an amino group. Likely, Asp119 makes critical hydrogen bonds to the 6-amino group and the N1 position of the enol form of 2-OHdATP, explaining the deleterious effect of the Asp119 mutations on the activity towards 2-OH-dATP.

Asp 120, a key residue in binding

Asp119 of MTH1 has been addressed extensively in the literature while the following residue, Asp120, has attracted less interest. The product complex of MTH1 reveals that Asp120 has a fundamental role in the recognition of the base. The MTH1 structure suggests that this is the most important and central amino acid in the recognition of the base. Asp120 is engaged in two hydrogen bonds with the base, one with N1 (2.9 $\AA$ ) and one with the 2-amino group ( $2.8 \AA$ ), (Figure $2 \mathrm{~A}$ ). 
The 2-amino group of 8-oxo-dGTP

Both Asn33 and Asp120 make hydrogen bonds to the 2-amino group of 8-oxo-dGMP, this position is central for the interaction. This is in agreement with studies of MTH1 substrate recognition using nucleotide analogs. Removing this group leads to a tenfold less efficient degradation [15].

\section{The 6-keto group of 8-oxo-dGTP}

The 6-keto group of 8-oxo-dGTP is not critical for the interaction with 8-oxo-dGTP. Its removal actually results in a substrate with slightly better affinity for MTH1 [22]. Removal of the 6-keto group and subsequent aromatization is accompanied by loss of the N1 hydrogen, making N1 a much better hydrogen bond acceptor. This will make the hydrogen bond interaction between N1 and Asp120 more favorable, Asp120 donates a hydrogen bond to N1 and the other oxygen of Asp120 accepts one hydrogen bond from the 2-amino group of 8 -oxo-dGTP. This is consistent with the observation that the enol forms of 2-OH-dATP and disoITP are very good substrates for MTH1 [22]. These nucleotides also lack a hydrogen in the N1 position and can therefore accept a hydrogen bond from Asp120.

\subsection{Preference for deoxyribonucleotides}

Interestingly, 8-oxo-dGTP is a considerably better substrate for MTH1 than 8-oxo-GTP [6]. The oxygen of the carbonyl of Thr8 makes a hydrogen bond (2.7 $\AA$ ) to the 3 hydroxyl-group of the 2-deoxyribose sugar. There are no possibilities for favorable 
interactions to the 2-hydroxyl-group of a ribose. The hydroxyl group would, on the contrary, be directed into a hydrophobic pocket composed of Leu9 and Val83, an unfavorable environment for this group. We suggest that this could explain the lower activity towards the ribonucleotide compared to the deoxyribonucleotide [6]. This difference in activity is not observed in Mut that has similar activity towards 8-OHdGTP and 8-OH-GTP [23].

\subsection{Recognition of the oxidized nucleotide}

To our surprise, the structure of the 8-oxo-dGMP complex revealed very few direct interactions between MTH1 and the 8-oxo position of the oxidized base. The closest contacts between the 8-oxo oxygen and MTH1 are to Met81 (3.3 $\AA)$ and Phe27 (3.5 $)$. The N7 hydrogen that accompanies the 8-oxo oxidation coordinates one water molecule but is not in direct contact with the protein. The N7 position is used by hOGG1 to discriminate between the oxidized and the unoxidized forms of the nucleotide [18]. Comparison of the apo structure and the product complex shows a slight movement of the $\varepsilon$-carbon of Met81 and insignificant change in the Phe27 position. The contacts of the N7 hydrogen and the 8-oxo group appear too weak to be responsible for the substrate specificity. The enzyme needs to discriminate between the oxidized and the unoxidized nucleotide. There is a 17-fold difference between the $\mathrm{K}_{\mathrm{M}}$ of dGTP and 8-oxo-dGTP [6]. A 17-fold difference corresponds to an approximate binding energy difference of 2.4 $\mathrm{kcal} / \mathrm{mol}$, roughly half the energy of a hydrogen bond. This energy difference is in a range that could be explained by preferred binding to the 6-enol-8-keto form of 8-oxodGTP (supplementary Figure S2). A hydrogen bond donating group, like an amino group 
or hydroxy (enol), in the 2 position appears to be very important for the overall affinity [15]. A hydrogen bond acceptor in the N1 position also appears important for binding [15]. We speculate that the 1- and 6-position is critical for the specificity and that the 6enol-8-keto form of 8-oxo-dGTP is able to donate and accept hydrogen bonds at these specific positions. The 8-oxo group of 8-oxo-dGTP makes the 6-enol form a more prominent and stable tautomer than for the un-oxidized nucleotide and suggests that significant levels of the 6-enol-8-keto form exists in solution [24]. The modification also significantly changes the electrostatic potential of the base [25]. We thus hypothesize that the basis for the difference in affinity between oxidized and un-oxidized nucleotide is that the 8-oxo modification of the base influences the keto-enol tautomerization and electrostatic properties of the opposite side of the base, which makes several key interactions with MTH1.

\section{Hydrogen bonding to the 6-enol-8-keto form of 8-oxo-dGTP}

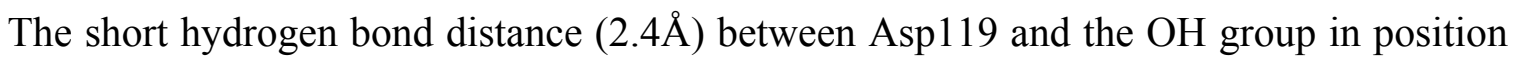
6 of the 6-enol-8-keto form of 8-oxo-dGTP (Figure 2A) supports the presence of this tautomer. The strong hydrogen bond implies an interaction between a negatively charged (deprotonated) carboxylate and an $\mathrm{OH}$ group. The observed hydrogen bonding pattern implicates that Asp120 is protonated, likely caused by effects of the local environment on its pKa. Binding of the alternative, 6-keto-8-keto tautomer would require that at least Asp119 is protonated. Thus, in both cases at least one of the carboxylate groups needs to be protonated. 


\subsection{Comparison of the NMR and crystal structures of MTHI}

The rmsd between the NMR structure and the crystal structure of MTH1 is as high as 1.8 $\AA$ [1]. The core beta sheet has the same structure but differences are present in several structural elements extending from the core sheet and surrounding the active site (Figure 3). There is a large difference in the position of Phe 27 and its loop between the NMR and crystal structures, Phe27 interacts with the product. Glu100 and neighboring residues also show significant differences between the crystal structures and the NMR structure, Glu100 is a metal coordinating residue that is essential for activity [1]. Two additional loops show significant main chain differences surrounding Gly76 and Gln142. It is possible that some of these differences may be explained by protein dynamics.

\subsection{Possibilities for structure based drug design}

It was recently demonstrated that MTH1 assists in eliminating RAS-induced oxidative damage to prevent induction of senescence [26]. Hence, any inhibitors of MTH1 may potentially increase the amount of RAS-induced oxidative damage and thereby make cancer cells enter pre-mature senescence leading to reduced cancer growth. This may be a very appealing strategy for treatment of cancer, also since the MTH1-/- knockout mice show very mild symptoms and only have limited inductions of tumors with very late onset. Thus, any MTH1 inhibitors are likely to have very few toxic effects to normal cells. The MTH1 structure presented in this study opens possibilities for structure based drug design for selectively targeting MTH1. 


\section{Acknowledgements}

This work was supported by grants from the Swedish Research Council (2010-5200), the Wenner-Gren Foundations and the Swedish Foundation for Strategic Research to P.S. The Swedish Research Council (2009-161; 2008-2655), Swedish Children's Cancer Foundation and the Swedish Pain Relief Foundation to T.H. The Swedish Cancer Society to T.H. and M.H. The authors thank Uwe Müller at BESSY for support at beamline BL14.1.

\section{References}

[1] Mishima, M. et al. (2004). Structure of human MTH1, a Nudix family hydrolase that selectively degrades oxidized purine nucleoside triphosphates. J Biol Chem $279,33806-15$.

[2] Nakabeppu, Y., Kajitani, K., Sakamoto, K., Yamaguchi, H. and Tsuchimoto, D. (2006). MTH1, an oxidized purine nucleoside triphosphatase, prevents the cytotoxicity and neurotoxicity of oxidized purine nucleotides. DNA Repair (Amst) 5, 761-72.

[3] Hori, M., Satou, K., Harashima, H. and Kamiya, H. (2010). Suppression of mutagenesis by 8-hydroxy-2'-deoxyguanosine 5'-triphosphate (7,8-dihydro-8-oxo2'-deoxyguanosine 5'-triphosphate) by human MTH1, MTH2, and NUDT5. Free Radic Biol Med 48, 1197-201.

[4] Tsuzuki, T. et al. (2001). Spontaneous tumorigenesis in mice defective in the MTH1 gene encoding 8-oxo-dGTPase. Proc Natl Acad Sci U S A 98, 11456-61. 
[5] Rai, P., Onder, T.T., Young, J.J., McFaline, J.L., Pang, B., Dedon, P.C. and Weinberg, R.A. (2009). Continuous elimination of oxidized nucleotides is necessary to prevent rapid onset of cellular senescence. Proc Natl Acad Sci U S A $106,169-74$.

[6] Fujikawa, K., Kamiya, H., Yakushiji, H., Nakabeppu, Y. and Kasai, H. (2001). Human MTH1 protein hydrolyzes the oxidized ribonucleotide, 2-hydroxy-ATP. Nucleic Acids Res 29, 449-54.

[7] Fujikawa, K., Kamiya, H., Yakushiji, H., Fujii, Y., Nakabeppu, Y. and Kasai, H. (1999). The oxidized forms of dATP are substrates for the human MutT homologue, the hMTH1 protein. J Biol Chem 274, 18201-5.

[8] Hayakawa, H. et al. (1999). Metabolic fate of oxidized guanine ribonucleotides in mammalian cells. Biochemistry 38, 3610-4.

[9] (1994). The CCP4 suite: programs for protein crystallography. Acta Crystallogr D Biol Crystallogr 50, 760-3.

[10] Long, F., Vagin, A.A., Young, P. and Murshudov, G.N. (2008). BALBES: a molecular-replacement pipeline. Acta Crystallogr D Biol Crystallogr 64, 125-32.

[11] Perrakis, A., Morris, R. and Lamzin, V.S. (1999). Automated protein model building combined with iterative structure refinement. Nat Struct Biol 6, 458-463.

[12] Emsley, P. and Cowtan, K. (2004). Coot: model-building tools for molecular graphics. Acta Crystallogr D-Biol Crystallogr 60, 2126-2132.

[13] Murshudov, G.N., Vagin, A.A. and Dodson, E.J. (1997). Refinement of macromolecular structures by the maximum-likelihood method. Acta Crystallogr D-Biol Crystallogr 53, 240-255. 
[14] Mildvan, A.S. et al. (2005). Structures and mechanisms of Nudix hydrolases. Arch Biochem Biophys 433, 129-43.

[15] Kamiya, H., Yakushiji, H., Dugue, L., Tanimoto, M., Pochet, S., Nakabeppu, Y. and Harashima, H. (2004). Probing the substrate recognition mechanism of the human MTH1 protein by nucleotide analogs. J Mol Biol 336, 843-50.

[16] Nakamura, T. et al. (2010). Structural and dynamic features of the MutT protein in the recognition of nucleotides with the mutagenic 8-oxoguanine base. J Biol Chem 285, 444-52.

[17] Fromme, J.C., Banerjee, A., Huang, S.J. and Verdine, G.L. (2004). Structural basis for removal of adenine mispaired with 8-oxoguanine by MutY adenine DNA glycosylase. Nature 427, 652-6.

[18] Bruner, S.D., Norman, D.P. and Verdine, G.L. (2000). Structural basis for recognition and repair of the endogenous mutagen 8-oxoguanine in DNA. Nature 403, 859-66.

[19] Bessman, M.J., Frick, D.N. and O'Handley, S.F. (1996). The MutT proteins or "Nudix" hydrolases, a family of versatile, widely distributed, "housecleaning" enzymes. J Biol Chem 271, 25059-62.

[20] Takahashi, M. et al. (2002). Role of tryptophan residues in the recognition of mutagenic oxidized nucleotides by human antimutator MTH1 protein. J Mol Biol $319,129-39$.

[21] Sakai, Y., Furuichi, M., Takahashi, M., Mishima, M., Iwai, S., Shirakawa, M. and Nakabeppu, Y. (2002). A molecular basis for the selective recognition of 2hydroxy-dATP and 8-oxo-dGTP by human MTH1. J Biol Chem 277, 8579-87. 
[22] Kamiya, H. et al. (2006). Recognition of nucleotide analogs containing the 7,8dihydro-8-oxo structure by the human MTH1 protein. J Biochem 140, 843-9.

[23] Taddei, F., Hayakawa, H., Bouton, M., Cirinesi, A., Matic, I., Sekiguchi, M. and Radman, M. (1997). Counteraction by MutT protein of transcriptional errors caused by oxidative damage. Science 278, 128-30.

[24] Venkateswarlu, D. and Leszczynski, J. (1998). Tautomeric equilibria in 8oxopurines: implications for mutagenicity. J Comput Aided Mol Des 12, 373-82.

[25] Aida, M. and Nishimura, S. (1987). An ab initio molecular orbital study on the characteristics of 8-hydroxyguanine. Mutat Res 192, 83-9.

[26] Rai, P., Young, J.J., Burton, D.G., Giribaldi, M.G., Onder, T.T. and Weinberg, R.A. (2011). Enhanced elimination of oxidized guanine nucleotides inhibits oncogenic RAS-induced DNA damage and premature senescence. Oncogene 30, 1489-96. 


\section{Figure legends}

Figure 1

(A) Enzymatic activity of MTH1 with 8-oxo-dGTP. (B) Overall ribbon representation of MTH1 in rainbow color representation, from the N-terminus (blue) to the C-terminus (red). 8-oxo-dGMP is shown in stick representation.

Figure 2

(A) A stereo representation of the binding of 8-oxo-dGMP to MTH1. Key hydrogen bonds in base recognition are displayed. 8-oxo-dGMP, residues coordinating 8-oxodGMP and metal coordinating residues are shown in stick representation. Waters in the vicinity of the active site is displayed as red spheres. Hydrogen bond distances are displayed.

(B) Overlay of the MTH1 and E.coli MutT 8-oxo-dGMP complexes illustrating the large differences in deoxyribose and base orientations as well as the similarity in the position of the $\alpha$-phosphate. 8-oxo-dGMP is shown in stick representation with green carbons from the MutT complex and gray carbons from the MTH1 complex.

\section{Figure 3}

An overlay of the C-alphas of the MTH1 complex crystal structure in blue and the MTH1 NMR structure in red. Loops with significant differences are indicated. E100, F27 and 8oxo-dGMP are shown in stick representation. 
Figure 1:
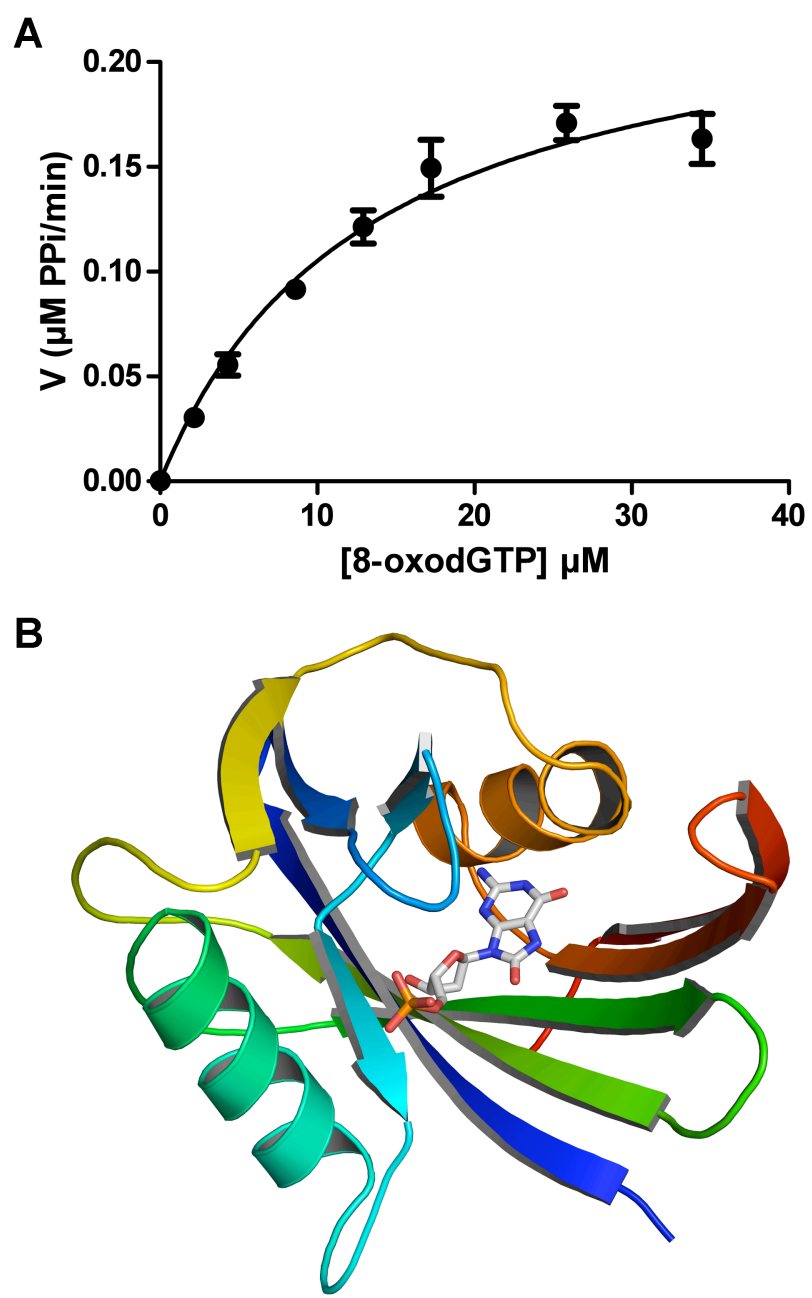
Figure 2:

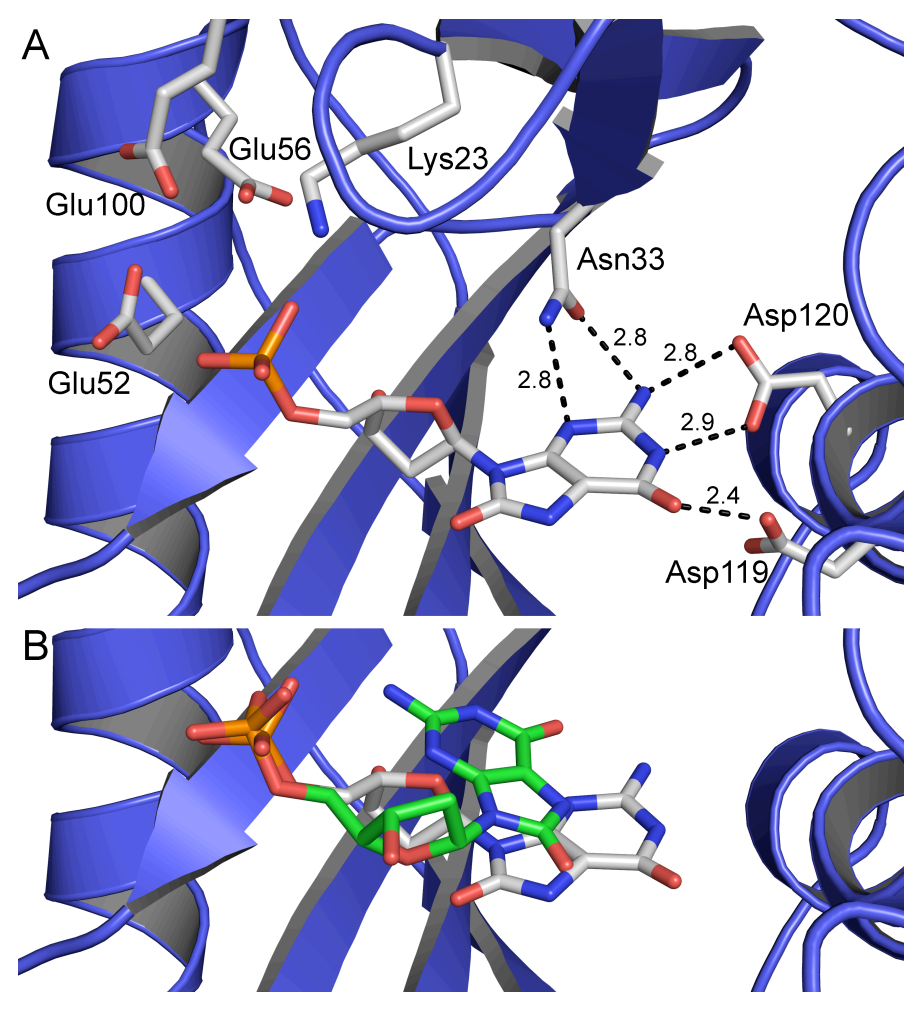


Figure 3:

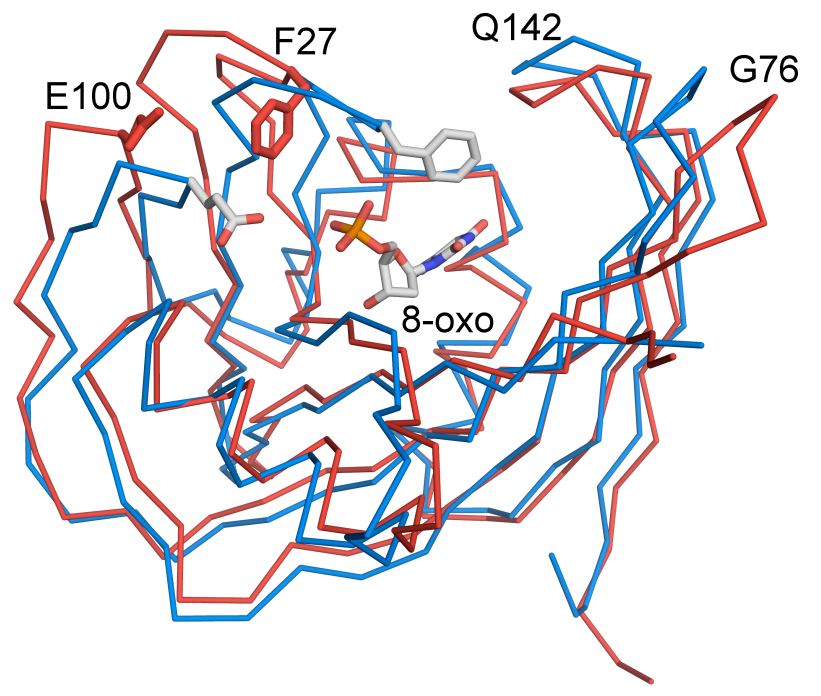


Table 1:

\begin{tabular}{lcc}
\hline \multicolumn{3}{c}{ Table1: Data collection and refinement statistics } \\
\hline & $\mathrm{Apo}$ & 8-oxo-dGMP \\
Space group & $\mathrm{P} 2{ }_{1}{ }_{1}{ }_{1}$ & $\mathrm{P} 2{ }_{1}{ }_{1}{ }_{1}$ \\
Cell parameters: & & \\
$\mathrm{a}, \mathrm{b}, \mathrm{c}(\AA)$ & $59.5,67.1,80.1$ & $59.5,67.3,80.0$ \\
$\quad \alpha, \beta, \mathrm{Y}\left({ }^{\circ}\right)$ & $90,90,90$ & $90,90,90$ \\
Resolution $(\AA)$ & $34-1.9(2.0-1.9)$ & $34-1.8(1.9-1.8)$ \\
Rmerge & $0.10(0.57)$ & $0.081(0.43)$ \\
Mn $(\mathrm{I} / \sigma(\mathrm{I}))$ & $11.6(3.3)$ & $12.2(3.3)$ \\
Completeness & $99.2(97.8)$ & $99.9(100)$ \\
Redundancy & $5.1(5.0)$ & $4.3(4.3)$ \\
Wavelength & 0.918 & 0.918 \\
$\mathrm{R}_{\text {work }} / \mathrm{R}_{\text {free }}(\%)$ & $19.3 / 24.5$ & $19.9 / 24.6$ \\
R.m.s. deviations: & & \\
$\quad$ Bond lengths $(\AA)$ & 0.022 & 0.024 \\
Bond angles $\left({ }^{\circ}\right)$ & 1.8 & 2.0 \\
Ramachandran zone & & \\
distribution $(\%)$ & $93.8 / 6.2 / 0 / 0$ & $93.4 / 6.6 / 0 / 0$ \\
\hline
\end{tabular}

